\title{
Enrollamiento en Conocoryphe heberti Munier-Chalmas \& Bergeron, 1889 (Cámbrico Medio, Cadena Ibérica, NE de España) y estructuras coaptativas en la familia Conocoryphidae
}

\author{
Enrolment in Conocoryphe heberti Munier-Chalmas \& Bergeron, 1889 \\ (Middle Cambrian, Iberian Chain, NE Spain) and coaptive structures \\ within family Conocoryphidae
}

\author{
J. Esteve ${ }^{1}$
}

\begin{abstract}
Enrolled Conocoryphe heberti is described with detail and compared with the conocoryphid Occatharia sdzuyi, both very abundant in the middle Cambrian from the Mediterranean subprovince. Both show different morphological novelties very useful to enrol and interlock the trilobite body. The morphological study allows us to recognize coaptative devices that show the possibility of a tight encapsulation enrolment. The coaptative devices are analyzed in cephalons, pigidia and almost completed outstretched trilobites of Bailiella levyi, Bailiaspis souchoni and Parabailiella languedocensis belonging to the same family. The coaptative structures analyzed show enough variability in cephalons and pigidia that allow suggesting several enrolment types within conocoryphids.
\end{abstract}

Key words: Trilobite, Conocoryphidae, coaptative structures, vincular depression, enrolment, Murero, Spain.

\section{RESUMEN}

Se describe por primera vez en detalle el enrollamiento de Conocoryphe heberti y se compara con el posible enrollamiento de Occatharia sdzuyi, ambas son especies de conocorífidos abundantes en el Cámbrico medio de la subprovincia Mediterránea. El análisis morfológico permite reconocer la presencia de una serie de estructuras coaptativas, relacionadas con el enrollamiento y que indican que estas dos especies poseerían una enrollamiento bastante hermético. Se analizan las estructuras coaptativas en cefalones, pigidios y ejemplares completos no enrollados de Bailiella levyi, Bailiaspis souchoni y Parabailiella languedocensis pertenecientes a la misma familia. Al analizar las estructuras coaptativas en cefalones y pigidios de estas cinco especies, se observa que estas estructuras presentan una cierta variabilidad dentro de su gran similitud, lo que permite proponer la posible existencia de varios tipos de enrollamiento dentro de los conocorífidos.

Palabras clave: Trilobites, Conocoryphidae, estructuras coaptativas, depresión vincular, enrollamiento, Murero, España.

\section{Introducción}

El enrollamiento es un comportamiento defensivo que se observa en distintos grupos de artrópodos. Este comportamiento en los trilobites se ha comprobado que ya se producía en el Cámbrico inferior; sin embargo, es mucho más común en los trilobites post-cámbricos que en los trilobites cámbricos. El mayor número de ejemplares enrollados hallados de trilobites post-cámbricos ha facilitado un mayor conocimiento y estudio del enrollamiento en los trilobites post-Cámbricos (Clarkson \& Henry, 1973; Henry, 1985; Speyer, 1985, 1988; Babcock \& Speyer, 1987; Hammann \& Rábano, 1987; Chatterton \&

\footnotetext{
1 Área y Museo de Paleontología. Dpto. CC. de la Tierra. Universidad de Zaragoza. E-50009 Zaragoza. Email: jorgeves@unizar.es
} 
Campbell, 1993; Bruton \& Haas, 1997; Clarkson \& Whittington, 1997; Bruton \& Nakrem, 2005). Aunque también existen ejemplos cámbricos, son menos conocidos por su menor abundancia en el registro fósil (Robison, 1964; Öpik, 1967; Stitt, 1983; BecqGiraudon \& Baillat, 2005; Esteve et al., 2008).

El desarrollo del enrollamiento como comportamiento y su posterior perfeccionamiento en los trilobites del Cámbrico se atribuye a dos hechos principales: protegerse ante la presencia de depredadores más eficaces (Bergström, 1973; Nedin, 1999), y como mecanismo de defensa ante avalanchas esporádicas de sedimento (Stitt, 1983; Babcock \& Speyer, 1987, Esteve et al., en prensa).

Tanto los trilobites cámbricos como los postcámbricos poseen estructuras coaptativas o de enrollamiento que les posibilitó enrollarse de distinta manera, lo que ha permitido también establecer una clasificación de los tipos de enrollamiento. En esta clasificación se distinguen básicamente dos tipos principales de enrollamiento según Bergström (1973): (i) Espiral, donde el pigidio se introduce por debajo del cefalón quedando así tapado. (ii) Esferoidal, donde el pigidio queda encajado en la parte anterior del cefalón, donde se puede encontrar un surco denominado vincular. Dentro de este último tipo se puede distinguir lo que Bergström (1973) definió como subtipo cilíndrico, en el que el trilobites no se cerraba perfectamente, quedando un hueco lateralmente. Con este último tipo de enrollamiento tendríamos a especies de trilobites muy comunes del Cámbrico como las pertenecientes al género Eccaparadoxides (Gil Cid, 1985; Becq-Giraudon \& Baillat, 2005).

En este trabajo nos vamos a centrar en el estudio de las estructuras coaptativas y de los posibles tipos de enrollamiento de cinco especies, pertenecientes a cinco género distintos, de la familia Conocoryphidae, siguiendo la propuesta para esta familia de Cotton (2001). La familia Conocoryphidae es típica del Cámbrico medio con una amplia distribución en la Provincia Acadobáltica sensu Sdzuy (1972) y en Siberia (Korobov, 1973; Egorova et al., 1982) y de manera mucho más esporádica se han encontrado algunos conocorífidos en China y el Himalaya (Zhang \& Jell, 1987; Jell \& Hughes, 1997). Dentro de esta amplia distribución, quizás la mayor diversidad y mayor rango de distribución se da en la Subprovincia Mediterránea sensu Sdzuy et al. (1999).

De todos modos a pesar de ser un grupo bastante abundante, son pocos los casos conocidos y figurados de ejemplares enrollados. Entre estos casos tenemos a la especie Conoryphe sulzeri (Schlotheim,
1823), que presenta un enrollamiento espiral (sensu Barrande, 1852; Šnajdr, 1958) o la especie Bailiella emarginata (Linnarsson, 1877) que también presenta este mismo tipo de enrollamiento y procedente del Cámbrico medio de la Cordillera Cantábrica tenemos Conocoryphe ovata Sdzuy, 1958 (Hernández Sampelayo, 1935, lám XVII, fig. 7).

Este trabajo tiene como principal objetivo describir el primer ejemplar de la especie Conocoryphe heberti enrollado y que permite reconocer para esta especie un enrollamiento de tipo «esferoidal». Este material procede de la localidad clásica de Murero en la provincia de Zaragoza, concretamente de la sección Rambla de Valdemiedes 1 (RV1; Liñán \& Gozalo, 1986). También describimos las estructuras coaptativas reconocidas en otras cuatro especies de distintos géneros de conocorífidos y la interpretación de su posible modo de enrollamiento; esta información es contextualizada dentro de la filogenia establecida para esta familia por Cotton (2001).

\section{Marco geológico y geográfico}

El ejemplar enrollado y gran parte del material estudiado para el análisis de las estructuras coaptativas procede de la localidad de Murero (provincia de Zaragoza en las Cadenas Ibéricas). Desde un punto de vista geológico, esta localidad se encuentra en la Unidad de Badules (fig. 1A), que es la prolongación de la zona Asturoccidental-Leonesa (sensu Gozalo \& Liñán, 1988).

En las distintas secciones realizadas en la Rambla de Valdemiedes al norte del pueblo de Murero, afloran materiales del Grupo Mesones y la base del Grupo Acón (Liñán \& Gozalo, 1986; Liñán et al., 2008). El Grupo Mesones se divide en tres formaciones: Valdemiedes, Mansilla y Murero. La edad de las misma va desde el Bilbiliense (parte alta del Cámbrico inferior) al Languedociense basal (parte alta del Cámbrico medio), esta misma edad presentan los materiales de la base del Grupo Acón que afloran en la Rambla de Valdemiedes (fig. 1B).

El espécimen de Conocoryphe heberti ha sido encontrado en el nivel 18 de la sección RV1 en la Formación Murero (fig. 1B). Esta formación consiste en una sucesión siliciclástica de lutitas verdes y marrones con intercalaciones de areniscas de grano muy fino y nódulos dispersos de carbonatos, depositados en un ambiente sublitoral (Liñán et al., 1993, 2002, 2008; Gozalo et al., 2003). La asociación de trilobites que hemos encontrado en el 

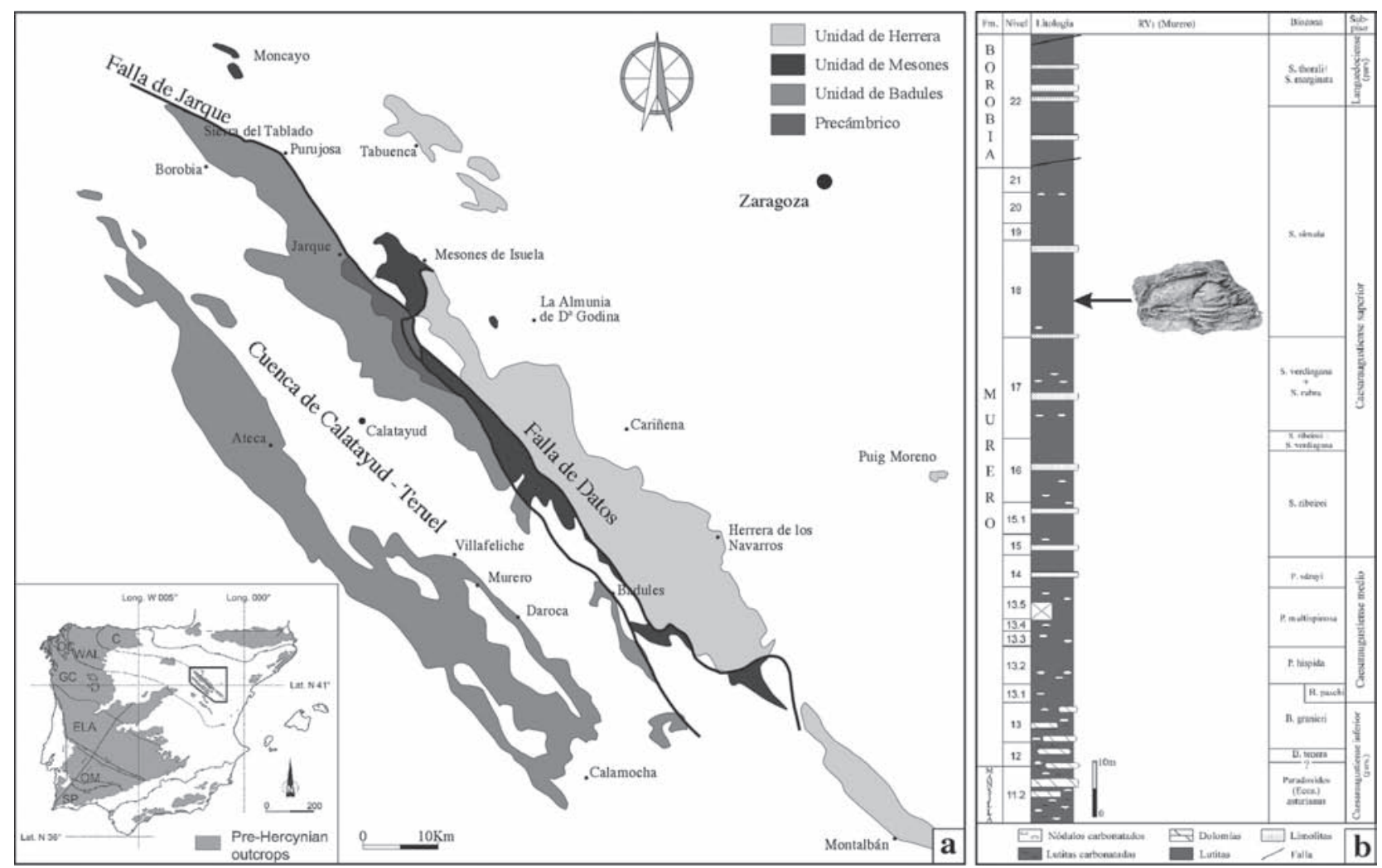

Fig. 1.-a, Mapa geológico de la Península Ibérica mostrando los yacimientos pre-Hercínicos y marco geológico de las Cadenas lbéricas (NE, España) y localización de los yacimientos de Murero. (Tomado de Liñán \& Gozalo, 1986 y Gozalo et al., 2008). b, Columna estratigráfica de la sección RV, con la situación estratigráfica de Conocoryphe heberti (Modificado de Liñán \& Gozalo, 1986).

mismo nivel, junto con este ejemplar es: Peronopsis ferox (Tullberg, 1880), Condylopyge rex (Barrande, 1846), Eccaparadoxides brachyrhachis (Linnarsson, 1883), E. pradoanus (Verneuil y Barrande, 1860), Solenopleuropsis rubra Sdzuy, 1958 y S. simula Sdzuy, 1958. Junto con estos trilobites también se encuentran equinodermos (Cincta y Eocrinoidea), hiolites, esponjas, braquiópodos y otros grupos problemáticos como gusanos. La asociación de trilobites nos sitúa a Conocoryphe heberti en la biozona de Solenopleuropsis simula en la parte alta del Caesaragustiense superior (Liñán \& Gozalo, 1986; Gozalo et al., 2003, 2008).

\section{Material y métodos}

Para este trabajo, como ya se ha indicado, la mayor parte del material estudiado procede de la sección RV1 en Murero, pero también hemos utilizado otros ejemplares, procedentes de diferentes yacimientos españoles y franceses. Parte de este material procede del yacimiento clásico de Los Barrios de
Luna (Cordillera Cantábrica) en los niveles basales de la Formación Oville, de edad Languedociense inferior (Sdzuy, 1961; Zamarreño, 1972), y de la Formación Coulouma en la Montaña Negra (S. de Francia), también del Languedociense inferior (Álvaro \& Vizcaïno, 1998).

A continuación se indica la procedencia del material utilizado en este estudio, donde se han analizado las estructuras coaptativas de las especies Conocoryphe heberti, Occataria sdzuyi, Parabailiella languedocensis, Bailiella levyi y Bailiaspis soucho$n i$, y las instituciones donde están depositados los especímenes junto con su número de registro.

De las Cadenas Ibéricas se dispone de un único ejemplar enrollado de Conocoryphe heberti (Munier-Chalmas \& Bergeron, 1889) procedente de la Formación Murero que se encuentra como molde externo del cefalón y tórax + pigidio y molde interno del cuerpo aplastado. Como material adicional de estudio, también procedente de la misma localidad, se han estudiado 19 cranidios y tres ejemplares con varios segmentos torácicos, dos de ellos semienrollados de Parabailiella languedocensis Thoral, 
Tabla 1.-Listado de términos tanto en castellano como inglés y abreviaturas utilizadas en las figuras

\begin{tabular}{lll}
\hline aam & Articulación en anillo medio & Half ring articulation \\
ap & Apodemas & Apodemes \\
cm & Conexiones marginales & Marginal conective \\
cpd & Campo posterior del pigidio delimitado & Demarcated posterior pigidial field \\
cpnd & Campo posterior del pigidio no delimitado & Non demarcated posterior pigidial field \\
$\mathrm{d}$ & Doblez & Doublure \\
$\mathrm{dv}$ & Depresión vincular & Vincular depression \\
eaa & Espina anillo axial & Spine of axial ring \\
$\mathrm{fa}$ & Faceta articular & Articulating facet \\
$\mathrm{fap}$ & Faceta articular pigidial & Pigidial articulatin facet \\
$\mathrm{p}$ & Pigidio & Pigidium \\
$\mathrm{pr}$ & Placa rostral & Rostral plate \\
$\mathrm{plr}$ & Pleura con terminación redonda & Rounded pleural termination \\
$\mathrm{plp}$ & Pleura con terminación en punta & Acute pleural termination \\
$\mathrm{pv}$ & Pestaña vincular & Vincular notch \\
$\mathrm{sc}$ & Sutura conectiva & Conective suture \\
$\mathrm{sf}$ & Sutura facial & Facial suture \\
$\mathrm{sp}$ & Surco pleural & Pleural furrow \\
$\mathrm{spi}$ & Surco pigidial & Pigidial furrow \\
\hline
\end{tabular}

1946; 21 cranidios y un ejemplar completo de Conocoryphe heberti y 12 cranidios de la subespecie Occataria sdzuyi courtessolei (Liñán \& Gozalo, 1986); depositados en el Museo Paleontológico de la Universidad de Zaragoza con las siglas desde MPZ 3148 hasta MPZ 3200 y MPZ 2009/1 hasta MPZ 2009/13. Todos ellos se encuentran conservados como moldes internos y externos, en lutitas verdes, marrones y rosadas.

Todo el material de la Zona Cantábrica procede de la localidad de Los Barrios de Luna, en la provincia de León, donde se encontró un ejemplar semienrollado de la especie Occatharia sdzuyi (Cortessole, 1967), más algunos ejemplares estirados casi completos, junto a cranidios y pigidios sueltos. También ha sido estudiado el holotipo de esta especie depositado en el Museo de Historia Natural de París con la sigla R09395. De esta misma localidad además se han estudiado siete cranidios, dos pigidios y dos ejemplares casi completos de la especie Bailiella levyi. Todos conservados como moldes externos e internos en lutitas marrones. El material figurado de esta localidad está depositado en el Museo Paleontológico de la Universidad de Zaragoza con las siglas MPZ 2009/14 hasta MPZ 2009/21.

Finalmente, como material de comparación complementario se han estudiado ejemplares completos de la especie Bailiaspis suchoni (Courtessole, 1967) depositados en la Colección de Paléontologie de l'Universite de Montperllier II con la sigla USTM-ECI01.

Todos los especímenes, tanto los moldes internos como los moldes externos, han sido estudiados mediante réplicas de látex que permiten observar las características de la anatomía de las caras externa e interna del exoesqueleto. Los látex se han estudiado bajo la lupa binocular (Leica@ M165C) y dibujados a cámara clara. Los ejemplares antes de ser fotografiados han sido blanqueados con cloruro de amonio para resaltar los detalles y se han fotografiado con una cámara Nikon@ ( F300. La terminología aplicada para las estructuras coaptativas o de enrollamiento es la traducción de la previamente usada por Speyer (1988), Chatertton \& Campbell (1993) y Clarkson \& Whittington (1997), también se ha definido algún término nuevo como depresión vincular, en la tabla 1 se ofrece un listado de estos nombres en castellano e inglés, así como las abreviaturas utilizadas en cada caso a la hora de señalarlas en las figuras.

\section{Descripción}

Aunque son escasos los ejemplares enrollados de conocorífidos, éstos han permitido describir y analizar las estructuras coaptativas presentes y su funcionalidad en el enrollamiento; por otro lado, una vez identificadas estas estructuras en estos ejemplares, ha sido posible reconocerlas y describirlas en otras especies de la familia, de las que hasta el momento no se conocen ejemplares enrollados. En primer lugar describiremos las estructuras coaptativas del ejemplar enrollado de Conocoryphe heberti y del semienrollado de Occatharia sdzuyi, para a continuación describirlas en las otras tres especies ya comentadas. 


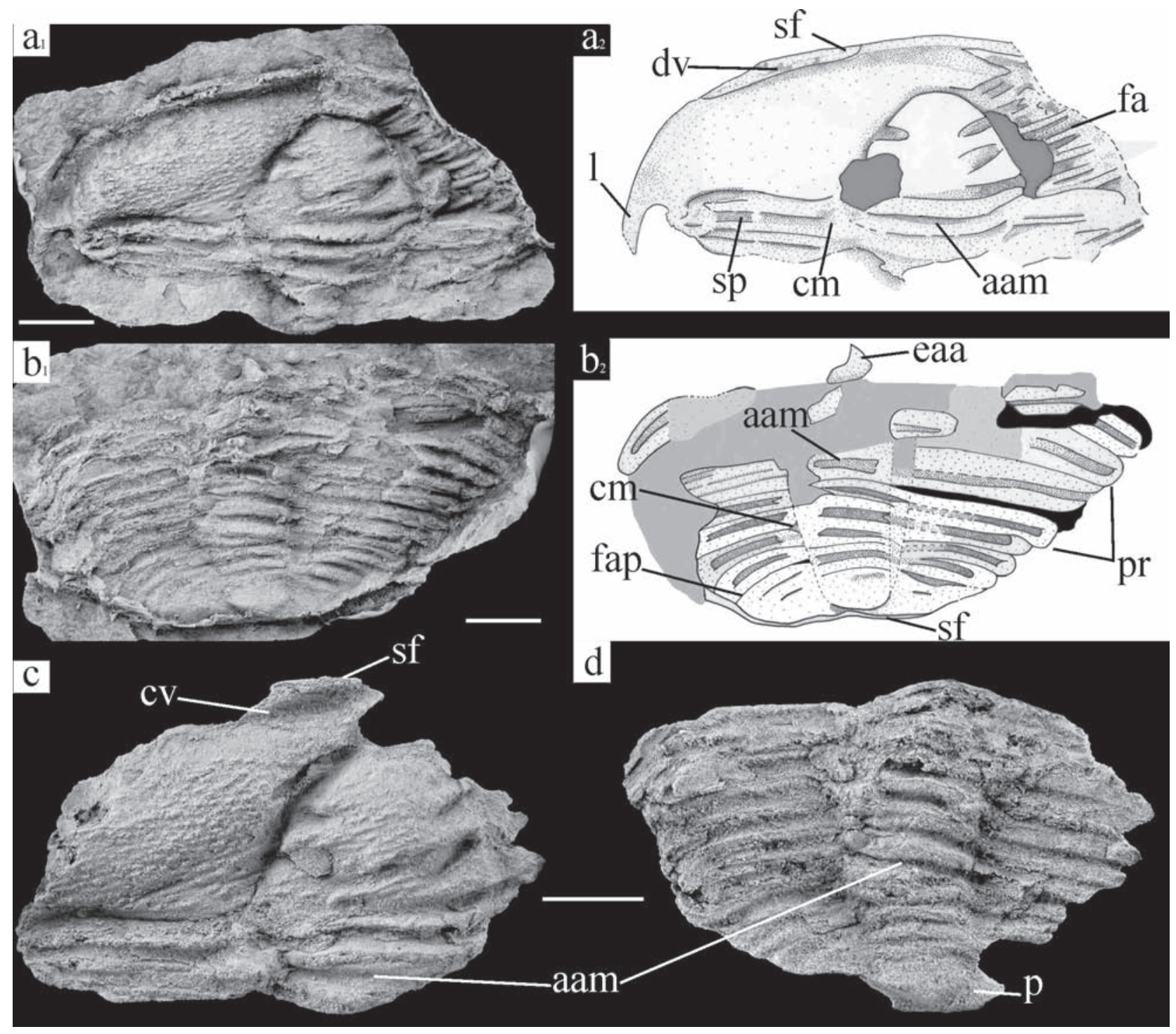

Fig. 2.-Conocoryphe heberti. (MPZ 2009/1) $\mathbf{a}_{1}$, Molde de látex en vista dorsal del cefalón más dos segmentos torácicos (MPZ 2009/1a). $\mathbf{a}_{2}$, Dibujo a cámara clara. $\mathbf{b}_{1}$, Molde de látex en vista dorsal del tórax más el pigidio (MPZ 2009/1b). $\mathbf{b}_{2}$, Dibujo a cámara clara. c. Molde interno en vista ventral del cefalón más dos segmentos torácicos (MPZ 2009/1c). d, Molde interno en vista ventral del tórax más pigidio (MPZ 2009/1c). (Escala $=2 \mathrm{~mm})$.

Conocoryphe heberti

(Munier-Chalmas \& Bergeron, 1889) (figura 2)

Observaciones: esta especie se encuentra descrita y con una amplia discusión en los trabajos de Thoral (1946), Sdzuy (1961), Courtessole (1973), Liñán \& Gozalo (1986) y Álvaro \& Vizcaïno (2003). Aquí simplemente vamos a destacar los elementos observables en las cuatro vistas (dos del molde interno y dos del molde externo) del ejemplar estudiado:
Látex de molde externo del cefalón (fig. $2 \mathrm{a}_{1}-\mathrm{a}_{2}$ ): se puede observar la vista dorsal del cefalón más los dos primeros segmentos torácicos. La fixígena derecha está rota, lo que nos permite ver, parcialmente, la cara ventral de las pleuras. En esta vista podemos observar las conexiones marginales en su vista dorsal, los surcos pleurales que terminan en el raquis, tanto por la cara dorsal como por la cara ventral. También se puede observar la faceta de articulación entre pleuras, que sería la que permitiría al trilobites enrollarse en vida. 


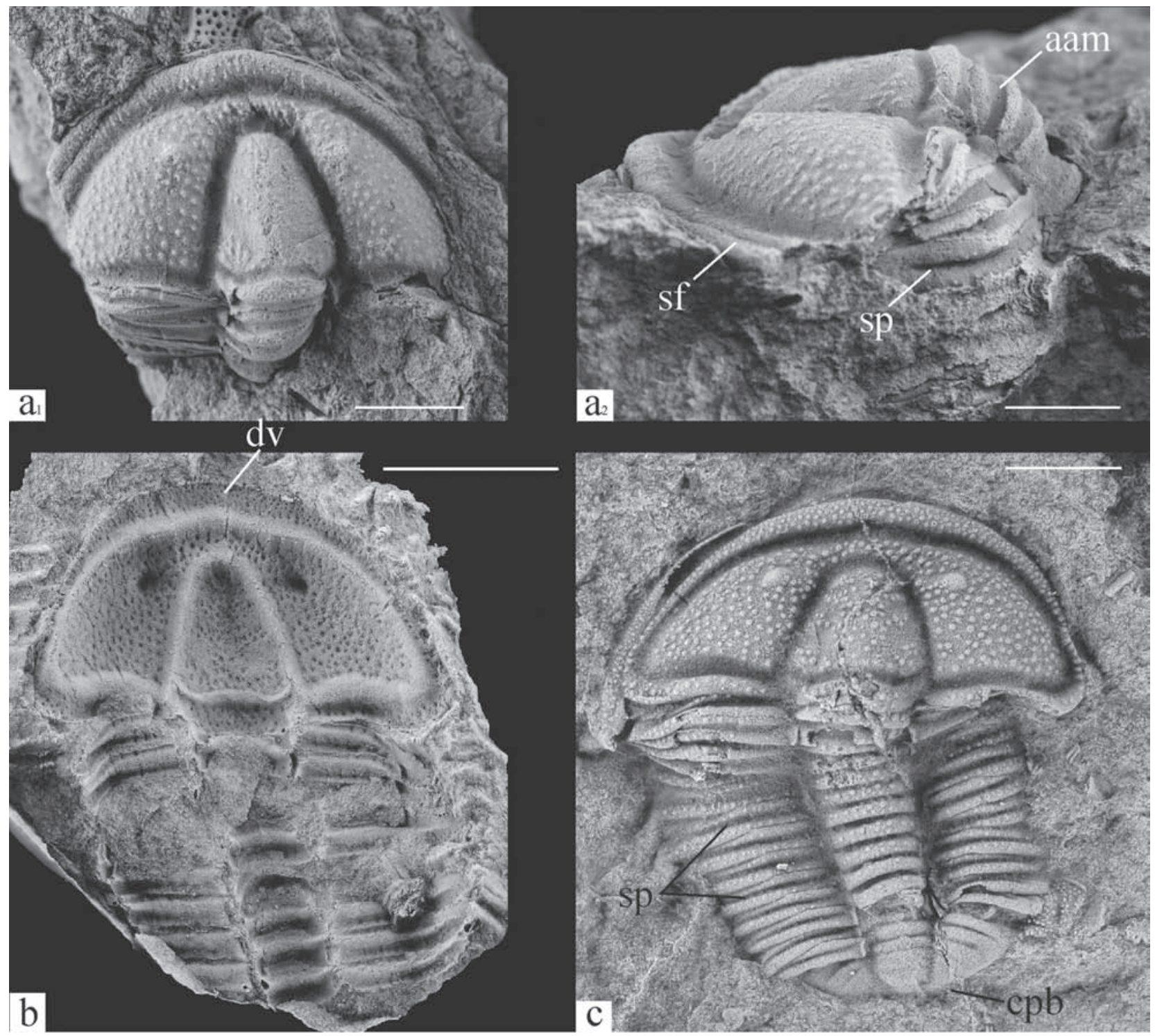

Fig. 3.-Occatharia sdzuyi. $\mathbf{a}_{1}$, Molde interno de la cara interna en vista ventral. $\mathbf{a}_{2}$, Molde interno en vista lateral. (MPZ 2009/14). b. Molde de látex de la cara interna en vista ventral (MPZ 2009/15). c, Molde de látex en vista dorsal. Holotipo (R09395). (Escala $\left.a_{1,2}=5 \mathrm{~mm}, \mathrm{~b}, \mathrm{c}=1 \mathrm{~cm}\right)$.

Látex del molde externo del tórax + pigidio (fig. $2 b_{1}-b_{2}$ ): el ejemplar muestra la mayor parte de los segmentos torácicos con los surcos pleurales que terminan en el raquis prolongándose en la articulación del anillo medio. Se puede observar cómo el pigidio y los segmentos pleurales se encuentran fijados a la depresión vincular, reforzándose el cierre mediante un pequeño saliente del cefalón.

Molde interno del cefalón (fig. 2c): igual que en el látex del molde externo (punto 1) tenemos el cefalón y los dos primeros segmentos torácicos. El cefalón se encuentra fragmentado, pero se puede observar la depresión vincular.

Molde interno del tórax + pigidio (fig. 2d): el molde interno muestra la mayor parte de los segmentos más el pigidio, igual que el látex del molde externo (punto 2). Los segmentos muestran unos surcos pleurales muy marcados, la articulación entre pleuras está también muy marcada. En el raquis se observa la articulación del anillo medio, que está poco desarrollada. 
Occatharia sdzuyi (Courtessole 1967)

(figuras $3 a_{1}-a_{2}$ )

Observaciones: en el caso de esta especie se han definido dos subespecies, O. sdzuyi sdzuyi (Courtessole, 1967) y $O$. sdzuyi courtessolei (Liñán \& Gozalo, 1986). Para una descripción y discusión destallada de ambas subespecies, véase Courtessole (1967, 1973), Liñán \& Gozalo (1986), Álvaro \& Vizcaïno (2003) y Álvaro (2007).

El espécimen estudiado es un molde interno de un ejemplar semienrollado, ya que no se observa que estén en contacto las pleuras torácicas con el cefalón y, por lo tanto, no se había cerrado totalmente el cuerpo del trilobites

El cefalón de esta especie es muy similar al de Conocoryphe heberti, también posee un abombamiento en el molde interno que correspondería a la depresión vincular aunque más ancha. La mayor diferencia encontrada en el cefalón sería la presencia de un par de protuberancias fixigenales. Además, se conservan tres segmentos torácicos, los cuales muestran unos surcos pleurales muy desarrollados (fig. $3 \mathrm{a}_{1}-\mathrm{a}_{2}$ ). Los surcos pleurales continúan en el raquis, donde se forma la articulación del anillo medio (fig. $3 \mathrm{a}_{2}$ ).

\section{Estructuras coaptativas en Conocoryphidae}

Los conocorífidos son una familia de trilobites del Cámbrico medio, revisados recientemente junto a otros pticopáridos ciegos por Cotton (2001), este autor incluye en esta familia los siguientes géneros y subgéneros: Conocoryphe Hawle \& Corda, 1847 (= Couloumania Thoral, 1946), Ctenocephalus (Ctenocephalus) Hawle \& Corda, 1847, Elyx Angelin, 1854, Bailiella Matthew, 1885, Ctenocephalus (Hartella) Mathew, 1885, Cainatops Matthew, 1889 (= Cornucoryphe Sdzuy \& Liñán, 1996), Bailiaspis (Bailiaspis) Resser, 1936, Parabailiella Thoral, 1946, Bailiaspis (Tchaispis) Korobov, 1966, posteriormente se añade a la familia el género Occatharia Álvaro \& Vizcaïno 2003.

Parabailiella considerado como género por Thoral (1946) y Cotton (2001) y otros autores lo consideran, por cuestiones utilitarias, como subgénero de Conocoryphe (Sdzuy, 1961 y posteriores), en este trabajo lo consideraremos a nivel de género. En el presente estudio no se han incluido los géneros Ctenocephalus, Cainatops y Elyx. Los dos primeros se han excluido por poseer un cefalón muy derivado respecto al resto de la familia; así, Cainatops presenta una larga espina en posición anterior (véase Sdzuy \& Liñán, 1996) o bien por poseer protuberancias en la parte anterior del cefalón como en el caso de Ctenocephalus, que se ha interpretado de varias formas, una de ellas como lugar donde se podrían alojar los huevos o la nidada (véase Fortey \& Hughes, 1998). Estos cefalones tan derivados seguramente implicarían un modo de enrollamiento distinto respecto al resto de los representantes de la familia. El tercer género, Elyx, se ha excluido por su distribución paleogeográfica, ya que no se ha encontrado en la Subprovincia Mediterránea.

Los conocorífidos, al igual que otros trilobites cámbricos, presentan adaptaciones en el cefalón, tórax y pigidio que les permitiría enrollarse, con más o menos efectividad y hermetismo. De estas adaptaciones, las estructuras coaptativas que presenta el cefalón son seguramente las más importantes a la hora de caracterizar los diferentes tipos de enrollamiento. Estas estructuras son observables tanto en las piezas del caparazón aisladas como en los trilobites completos, tanto enrollados como no, pudiéndose inferir, a partir de ellas, cuál sería el tipo de enrollamiento más probable (Bergström, 1973; Stitt, 1983).

\section{Cefalón}

Aunque la morfología del cefalón es muy similar en la mayoría de los conocorífidos (Cotton, 2001; Kim et al., 2002), la disposición general del borde anterior del cefalón presenta características que en algunos casos son consideradas de nivel genérico y en otros de nivel específico; además, estas diferencias, seguramente, también harán que varíe la forma de enrollarse de los distintos taxones. De la morfología del cefalón de los conocorífidos podemos inferir estructuras coaptativas, sobre todo analizando sus moldes internos.

En la cara interna de la parte anterior del cefalón, los conocorífidos presentan una estructura homóloga al surco vincular en la doblez del cefalón, para el que se ha acuñado en este trabajo el término depresión vincular (figs. 2, 3a-c, 4a-b, 5a-c 7a-b, 8a-f), ya que el término surco hace referencia a una estructura más estrecha y que mantiene su anchura prácticamente constante en todo el cefalón, como por ejemplo el caso de Phacops (Speyer, 1988; Bruton \& Haas, 1997). Aunque este surco vincular es una estructura mucho más estrecha que la depresión 


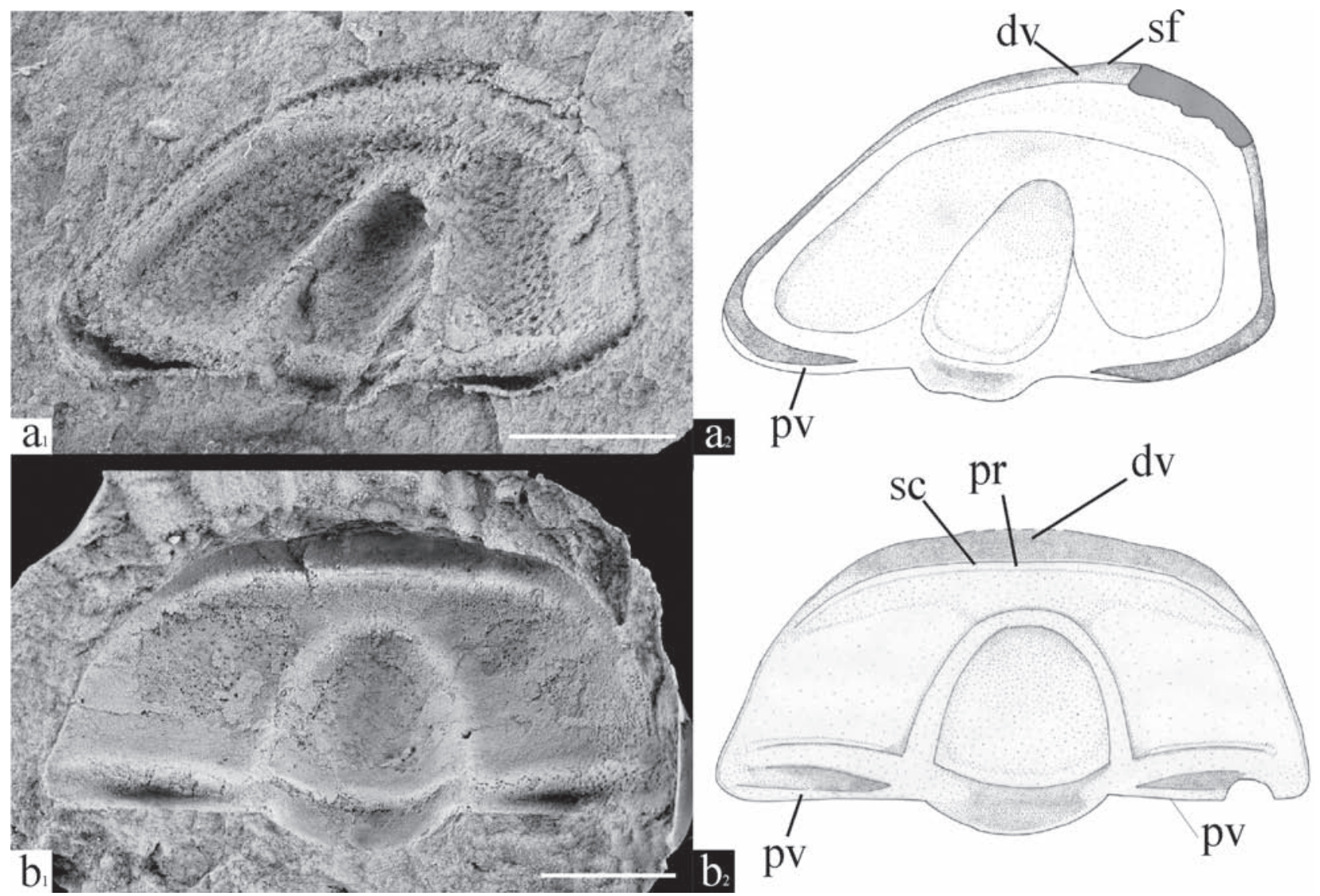

Fig. 4.-a, Conocoryphe heberti (MPZ 2009/2). $\mathbf{a}_{1}$, Molde de látex de la cara interna en vista dorsal, $\mathbf{a}_{2}$, Dibujo a cámara clara. b, Bailiella levyi (MPZ 2009/16). $\mathbf{b}_{1}$, Molde de látex de la cara interna en vista dorsal. $\mathbf{b}_{2}$, Dibujo a cámara clara. (cv, depresión vincular; d, doblez; pr, placa rostral, sf surco de fijación). (Escala = $5 \mathrm{~mm}$ ).

que presentan los conocorífidos, funcionalmente serían equivalentes: permitir encajar el pigidio y las puntas pleurales de los últimos segmentos, dándole estabilidad cuanto el trilobites esté enrollado. Esta depresión vincular presenta una geometría variable entre los distintitos géneros de conocorífidos, pero suponemos que su posible función en el enrollamiento era similar en todos ellos.

En la región ventral, por detrás de la depresión vincular situada en la doblez del cefalón los conocorífidos portan la placa rostral (Whittington, 1988) fuertemente fusionada a la doblez del cefalón (fig. 8e, f). La placa rostral presenta también distintos tamaños entre los conocorífidos (fig. 8), pero siempre se trata de una zona sobreelevada de la parte posterior del doblez del cefalón (fig. 8e, f). La placa rostral conectaría, mediante tejido blando, el exoesqueleto con el hipostoma (Fortey, 1990). Además, tendría la función de actuar de tope cuando se enrollara el trilobites. Por último, los cranidios de los conocorífidos poseen unas pestañas vinculares en la parte posterior, que servirían a los trilobites para enganchar las primeras pleuras durante el enrollamiento (fig. 4a, b). El tipo de enrollamiento efectuado por los distintos géneros de conocorífidos dependerá, en gran medida, de la geometría y de la posición de la depresión vincular y de la placa rostral.

Entre los conocorífidos podemos encontrar distintas morfologías en los bordes anteriores de sus cranidios

- Bailiella levyi (Munier-Chalmas \& Bergeron, 1889), presenta una depresión vincular muy profunda e inhomogénea (más ancha sagitalmente y estrecha exsagitalmente), y una placa rostral muy estrecha (1/3 de la anchura máxima de la depresión vincular, fig. 8a). El pigidio podría quedar encajado en esta depresión, lo que permitiría seguramente un tipo de enrollamiento pseudo-esferoidal, ya que pigidio y cefalón no quedarían alineados pero tam- 


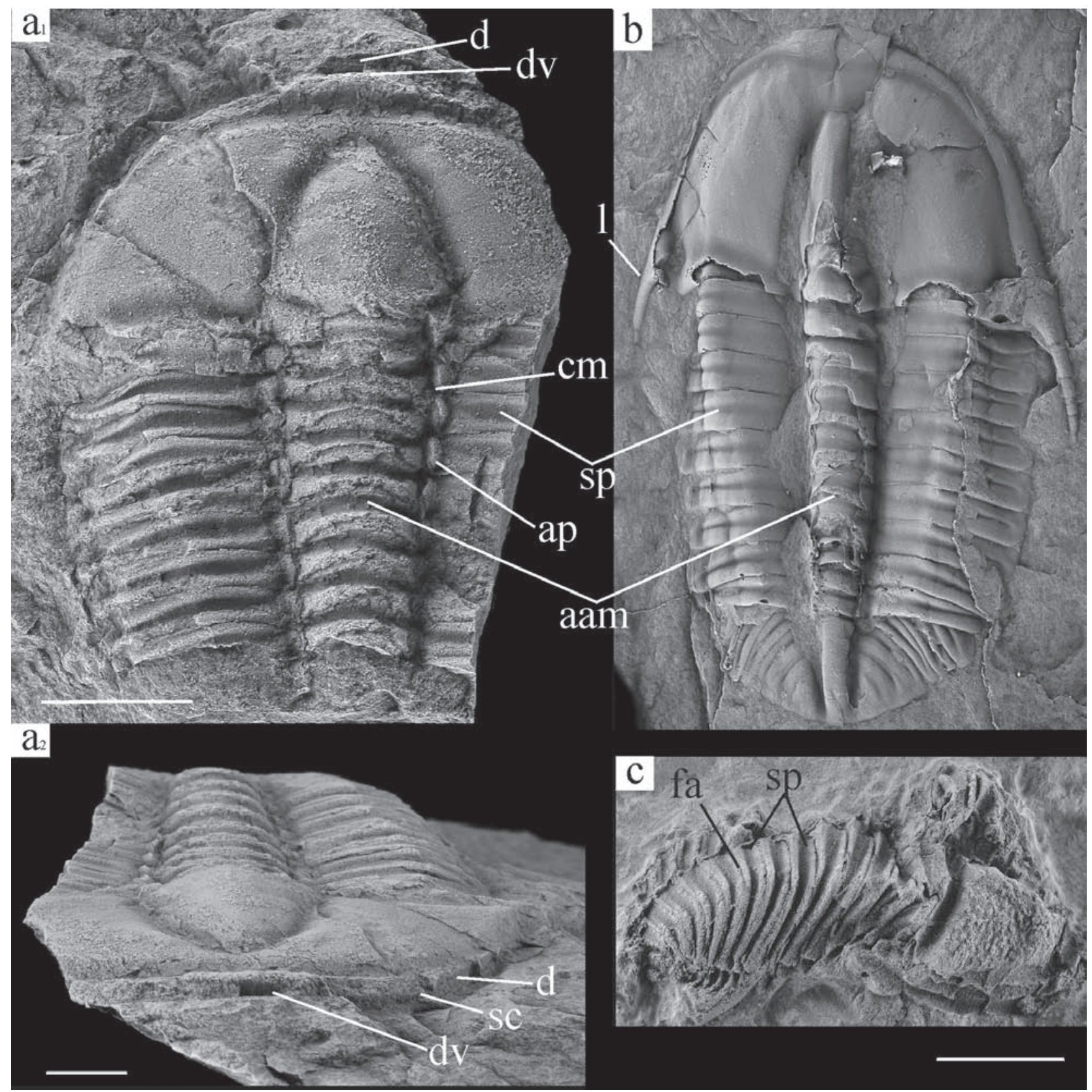

Fig. 5.-a, Bailiella levyi (MPZ 2009/6). $\mathbf{a}_{1}$, molde de la cara interna en vista ventral, con el canalón roto mostrando la doblez y el interior de la cuenca vincular. $\mathbf{a}_{2}$, Vista frontal mostrando la sutura conectiva y la doblez del cefalón. b, Bailiaspis souchoni (USTMECI01), molde de látex en vista dorsal. c, Parabailiella cf. languedociensis (MPZ 2009/7), molde interno en vista lateral, espécimen semienrollado. (Escala $a_{1}, b=1 \mathrm{~cm}, a_{2}, c=5 \mathrm{~mm}$ ).

poco quedarían totalmente cubiertos por el cefalón. Linnarson (1877, p. 368) citó el enrollamiento en B. emarginata (Linnarsson, 1877), que según la clasificación actual se podría considerar como un enrollamiento espiral, lo que viene corroborado por la presencia de una depresión vincular ancha y ligeramente inhomogénea.
- Parabailiella languedocensis Thoral, 1946, presenta una depresión vincular profunda y muy inhomogénea, la placa rostral sería del mismo tamaño o ligeramente más ancha que la depresión vincular (fig. 8b). Con esta morfología el pigidio y parte de los últimos segmentos torácicos podrían quedar encajados en la depresión vincular, siendo cubiertos 


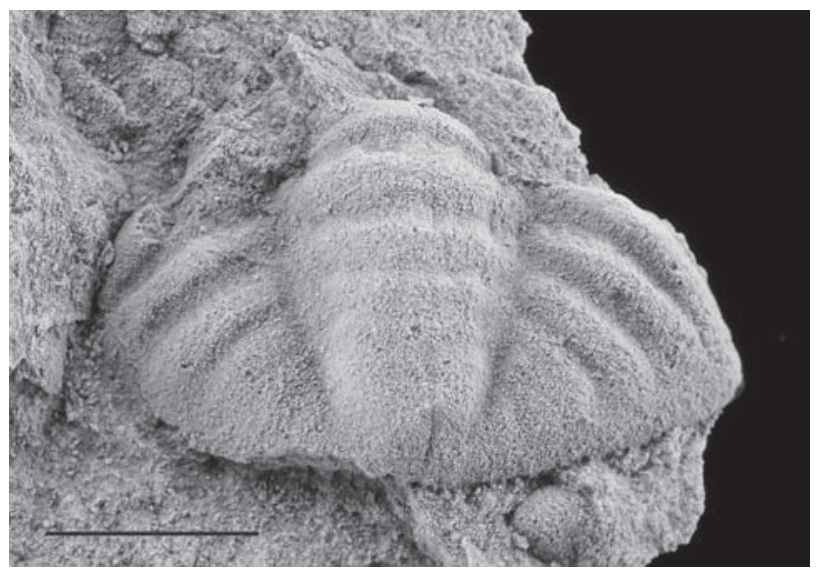

Fig. 6.-Molde interno del pigidio de Bailiella levyi (MPZ 2009/7) $($ Escala $=5 \mathrm{~mm})$.

por completo por el cefalón, dando como resultado más probable un tipo de enrollamiento espiral.

- Occatharia sdzuyi (Courtessole, 1967), posee una depresión vincular poco profunda y del mismo tamaño que la placa rostral (fig. 8c). A diferencia que los conocorífidos anteriores la depresión vincular termina en un pequeño reborde de menos de $1 \mathrm{~mm}$ de ancho (fig. $3 \mathrm{~b}$ ), que daría robustez al borde anterior.

- Conocoryphe heberti (Munier-Chalmas \& Bergeron, 1889), proporcionalmente posee la depresión vincular más estrecha de los conocorífidos aquí analizados. Ésta mantiene su anchura de forma más o menos constante en todo el perímetro del cranidio, la placa rostral es de dos a tres veces su anchura (Whittington, 1988). En el borde de la depresión vincular tenemos también una pequeña muesca de 1 a $2 \mathrm{~mm}$ de anchura denominada surco de fijación (figs. $2 \mathrm{a}_{2}, \mathrm{c}, 4 \mathrm{a}_{2}$ ). A lo largo de toda esta depresión se fijaría el pigidio y las puntas pleurales. Por tanto, debido a esta morfología, el borde del pigidio queda bien encajado contra el borde del cefalón «margen contra margen», quedando ambos alineados y dando así un tipo de enrollamiento esferoidal (fig. 2b).

- Bailiaspis souchoni (Courtessole, 1967), el borde anterior presenta un plectrum (sensu Kim et al., 2002), que es una extensión posterior del borde. Como resultado Bailiaspis posee una depresión muy inhomogénea (fig. 5b). Esta depresión tan ancha hace que el pigidio y parte de los segmentos torácicos encajen necesariamente por debajo del cefalón, por lo que nos encontraríamos, muy probablemente, ante un conocorífido con un enrollamiento de tipo espiral.

\section{Tórax}

Los conocorífidos presentan una morfología torácica característica y muy similar en los géneros de la familia. En el tórax se pueden observar estructuras coaptativas tanto en las pleuras como en el raquis. En las primeras, justo a la región más próxima al raquis, forman unos mecanismos de enganche, denominados conexiones marginales (sensu Bergström, 1973). Las pleuras están divididas en dos partes, una faceta de articulación y una banda pleural anterior, separadas mediante un surco pleural (fig. $5 a_{1}$, b). El surco pleural se extiende hacia fuera hasta llegar a la faceta pleural donde desaparece (figs. 3c, 5a, b). Se tata de un surco muy recto y bastante profundo lo que produce una buena delimitación de las bandas pleurales anterior y posterior (fig. 5c). La forma terminal de la pleura varía entre los distintos representantes de la familia, desde mas o menos redondeada en $B$. souchoni (fig. 5b) hasta una terminación más aguda en $C$. heberti (fig. 7a), aunque son sólo las primeras pleuras la que presentan esta modificación. Entre los distintos anillos del raquis, se encuentra una articulación denominada articulación del anillo medio, que sería la que se encargada de transmitir el movimiento cuando el trilobites se enrollaba (figs. $2 b_{2}, c, 3 a_{2}, 5 a_{1}, a_{2}$ ).

\section{Pigidio}

El pigidio de los conocorífidos es de un tamaño pequeño, usual entre pticopáridos ciegos (Cotton, 2001). A grandes rasgos los conocorífidos poseen un pigidio con una forma subtriangular con el ápice más o menos redondeado. Una diferencia importante a la hora del enrollamiento es la terminación del raquis. Éste puede acabar bien en una posición relativamente proximal sin observarse una clara delimitación entre el raquis y el campo posterior del pigidio (figs. $2 b_{1}, b_{2} ; 3 c ; 7 a ; 9 b$ ), dejando un campo posterior de una cierta amplitud, o bien el raquis perfectamente delimitado y que termina justo en el borde, sin apenas campo posterior (figs. $5 \mathrm{~b} ; 7 \mathrm{~b} ; 9 \mathrm{c}$ ). Otra diferencia entre los pigidios de conocorífidos es el ángulo de terminación formado mediante una secante desde el ápice. Este ángulo puede ser bien pequeño entre 8 y $15^{\circ}$ (fig. $2 \mathrm{~b}, 3 \mathrm{c} ; 6.7 \mathrm{a}$; 9d) o bien ángulos más abiertos entre 16 y $21^{\circ}$ (figs. 5b; 7b; 9a). En el caso con un raquis terminando bruscamente tenemos a Conocoryphe heberti o de Occatharia $s d z u y i$, igual que ocurre en algunas especies de 


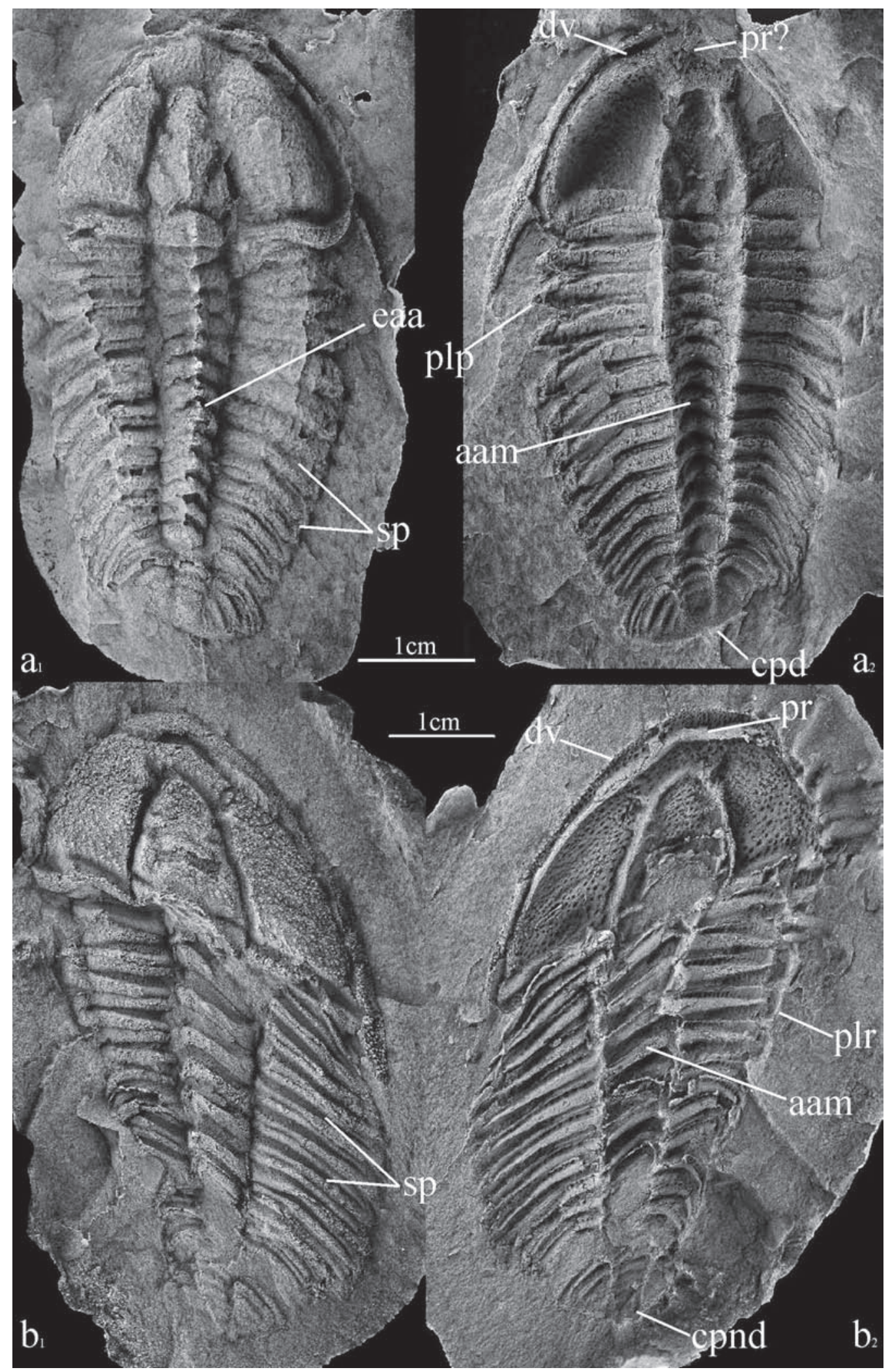

Fig. 7.-a, Conocoryphe heberti (MPZ 2009/8). $\mathbf{a}_{1}$, Molde de látex en vista dorsal. $\mathbf{a}_{2}$, Molde de látex de la cara interna en vista ventral. b, Parabailella languedociensis (MPZ 2009/9). $\mathbf{b}_{1}$, Molde de látex en vista dorsal. $\mathbf{b}_{2}$, Molde de látex de la cara interna en vista ventral. 
a
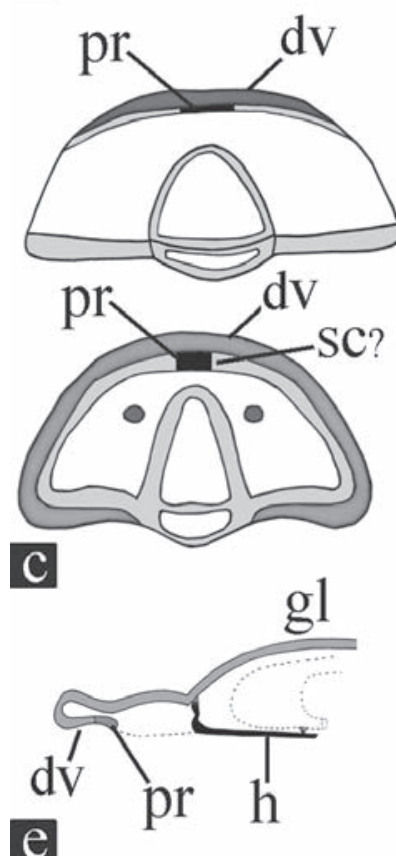
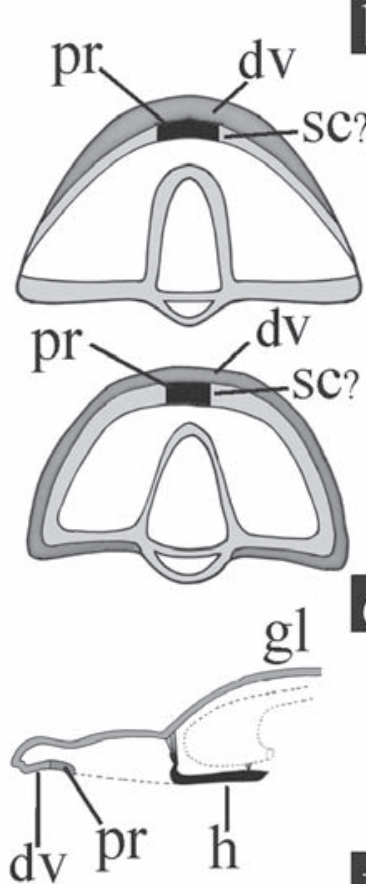

.

Fig. 8.-a-d, Reconstrucción de la cara ventral de los cranidios sin el hipostoma (a partir de ejemplares estudiados y recons trucciones de Šnajdr, 1958 y Whittington, 1988). a, Baililla levyi. b, Parabailiella languedociensis. c, Occatharia sdzuyi. d, Conocoryphe heberti. e-f, Reconstrucción en sección longitudinal del cefalón mostrando la relación de la depresión vincular y la placa rostral. (Reconstrucción de la posición del hipostoma tomada de Fortey, 1990). e, Parabailiella languedociensis. f, Conocoryphe heberti.

Bailiellas como en B. emarginata. En el caso donde el raquis termina suavemente tenemos a Bailiaspis souchoni, Parabailiella languedocensis o Bailiella levyi (figs. 5b, 6, 7b, 9), aunque en el caso de esta última, el ángulo es más abierto que en Bailiaspis y Parabailiella.

\section{Enrollamiento en los conocorífidos}

Los pticopáridos ciegos son un grupo de trilobites polifiléticos (Fortey, 1990; Cotton 2001) formado por cuatro clados. Los representantes de la familia Conocoryphidae constituyen un clado monofilético de acuerdo con Cotton (2001, p. 178). Las relaciones filogenéticas propuestas por Cotton (2001) entre los distintos géneros de conocorífidos son de gran utilidad a la hora de analizar cómo se adquieren las estructuras coaptativas aquí descritas a lo largo de los distintos clados. Sin embargo, aún no están bien resueltas las relaciones entre algunos
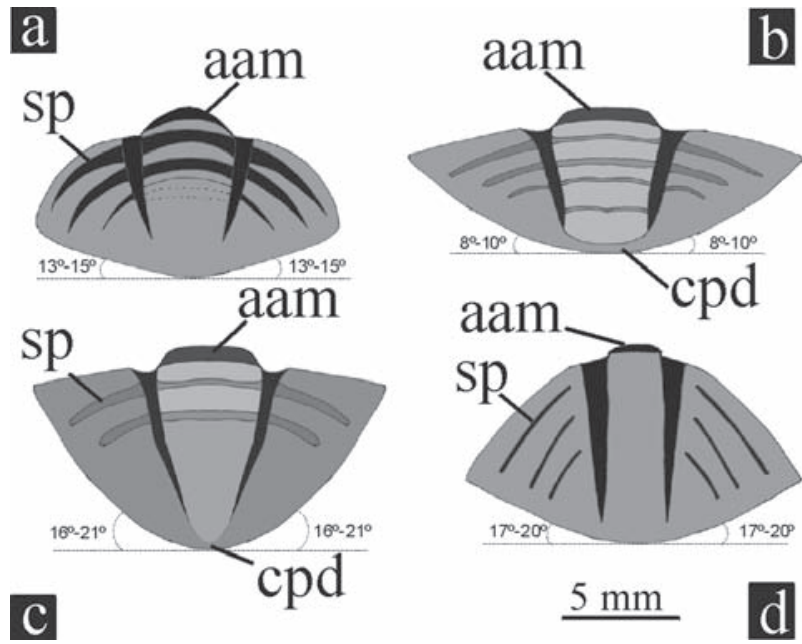

Fig. 9.-Reconstrucción de la cara dorsal de los pigidios. a, Bailiella levyi. b, Conocoryphe heberti c, Parabailiella languedociensis. d, Bailiaspis souchoni.

miembros de la familia. Este problema se debe a la imposibilidad de codificar muchos de los caracteres de las especies y, por otro lado, en qué grupo externo utilizar para enraizar el árbol filogenético.

El tipo de enrollamiento que presentan los distintos géneros de conocorífidos está íntimamente ligado con la morfología que presentan, tanto el cefalón y como el pigidio. El análisis realizado permite diferenciar dos tipos de cefalones en función de la depresión vincular:

1. Inhomogénea (más ancha en su parte central, fig. 8a, c).

2. Homogénea (igual anchura, fig. 8b, c).

Dos tipos de pigidios:

1. Campo posterior más o menos ancho por una atenuación de la parte posterior del raquis y ángulos abiertos.

2. Sin campo posterior, con el raquis bien definido y con ángulos más agudos.

El análisis morfológico de los cefalones y los pigidios de distintas especies muestra una correspondencia entre los dos tipos de depresiones vinculares y los dos tipos de pigidios. Se puede tener formas con depresión vincular inhomogénea y pigidios sin terminaciones bruscas y ángulos más agudos o bien formas contrarias con depresiones vinculares homogéneas con pigidios con campo posterior y ángulos más abiertos; estas dos configuraciones nos darían las formas extremas para el grupo estudiado. Sin embargo, no se trata de una relación fija y pode- 
mos encontrar variaciones intermedias entre ambas formas extremas. Así, en el caso de especies como Conocoryphe heberti en el extremo de depresiones vinculares homogéneas, con pigidios con campo proporcionalmente amplio y ángulos bastante abiertos, lo que facilita que el pigidio se fije en la depresión vincular, y de manera opuesta especies como Bailiaspis souchoni, donde el borde anterior presenta un plectrum y el raquis termina casi en el borde de forma muy suave y con un ángulo más agudo, lo que le permite encajar mejor en el plectrum.

Estas morfologías encontradas en los cefalones y pigidios de distintos conocorífidos podrían indicarnos diferentes tipos de enrollamiento para las distintas especies seleccionadas, y por lo tanto para los distintos géneros. Observamos enrollamientos esferoidales en las morfologías que presenta $C$. heberti y se deducen enrollamientos de tipo espiral en la morfología que observamos en Bailiaspis souchoni; pero también casos intermedios como $O$. sdzuyi y $B$. levyi con un enrollamiento pseudo-esferoidal aunque hay especies de Bailiella que podían presentar un enrollamiento de tipo espiral como es el caso de Bailiella emarginata, ya que presenta una depresión vincular más ancha (Westergård, 1936, p. 58, lám. XI, 2a). No es extraño que dentro de una misma familia, e incluso dentro de un mismo género, se encuentren tipos de enrollamientos distintos o variaciones de un tipo concreto (Fortey \& Owens, 1979; Hammann 1985; Chatertton \& Campbell, 1993; Esteve et al., en prensa).

A partir del cladograma desarrollado por Cotton (2001, p. 177) para Conocoryphidae (fig. 10), vemos que las estructuras coaptativas analizadas en distintos géneros y especies se pierden o se adquieren a lo largo de las ramas del árbol, tratándose en algunos casos de caracteres con un alto grado de convergencia evolutiva (por ej., el ángulo del pigidio). Examinando el desarrollo de los caracteres a lo largo del árbol comprobamos que la morfología de la depresión vincular es un carácter multiestado. La depresión vincular inhomogénea (carácter $\mathrm{C} 1$ ) es un carácter plesiomórfico mientras que la depresión vincular homogénea (carácter $\mathrm{C} 2$ ) es un carácter adquirido por el género Conocoryphe, o como el caso del plectrum (carácter C3) que se trata de un carácter sinapomórfico de Bailiaspis y del subgénero Tchaiaspis omitido del cladograma para simplificarlo. O también en el caso de Bailiella levyi una depresión aunque inhomogénea muy marcada y próxima al margen anterior (carácter $\mathrm{C} 4$ ). Por su lado, los caracteres analizados para el pigidio, como

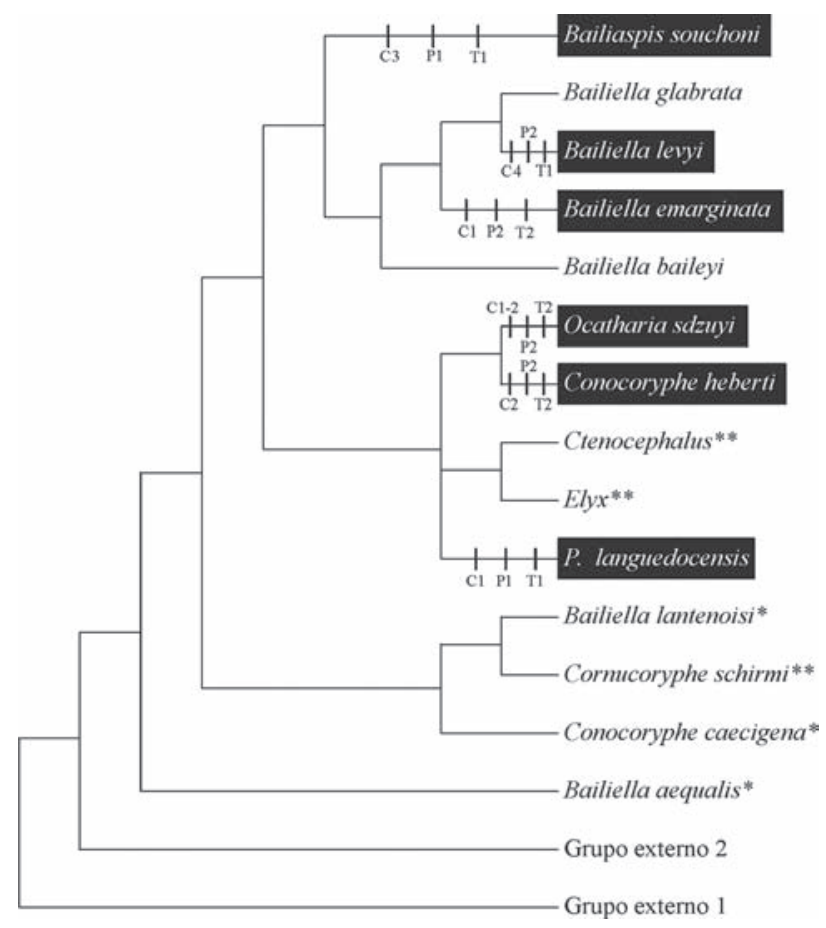

Fig. 10.-Árbol consenso majority-rule (Según Cotton, 2001). * Especies pendientes de un estudio taxonómico en profundidad. ** Especies fuera del presente estudio por poseer un cefalón muy derivado o no pertenecer al mismo paleocontinente.

es el ángulo, también varían desde formas agudas (carácter P1) a formas laxas (carácter P2) siendo éste un carácter convergente a lo largo de las ramas. Por último la terminación brusca (carácter T1) o suave (carácter T2) es sinapomórfico en el clado de Occatharia + Conocoryphe y autapomórfico en el caso de Bailiella emarginata.

Los análisis de las distintas estructuras coaptativas pueden ser útiles a la hora de poder caracterizar especies de los géneros: Bailiella, Conocoryphe y Parabailella. Cotton (2001, p. 193) ya discutió esta problemática, aunque basándose en otros caracteres; uno de los que le ofrecía una mayor consistencia era la «posición de la sutura facial con respecto al surco lateral (en el borde en Conocoryphe y cortando al surco en Bailiella y Parabailella)», para Cotton (op. cit.) este criterio indicaría que Conocoryphe y Parabailiella serían géneros distintos y no subgéneros. Este carácter de la posición de la sutura está íntimamente ligado con la posición y forma de la depresión vincular. Cuando la depresión vincular se corta por la sutura, tenemos depresiones muy inhomométricas [Bailiella y Parabailella (fig. 7a, b)]. Bailiaspis sería un caso extremo de enrollamiento que va de forma espiral pura a pseudo-esferoida- 
les, pero dicha morfología no podría dar enrollamientos esferoidales. Por el contrario, cuando la sutura no corta el surco, la depresión vincular puede variar entre formas menos inhomométricas (Occatharia, fig. $7 \mathrm{c}$ ) a formas más homogéneas (Conocoryphe, fig. 7d).

En el caso de Bailiaspis y Bailiella, Kim et al. (2002, p. 826), proponen la presencia de un plectrum en Bailiaspis para diferenciar ambos géneros. Igualmente la morfología de esta invaginación (el plectrum) permite suponer que su función pudiera ser facilitar la fijación del pigidio bajo el cefalón, dando un enrollamiento espiral. Mientras que en Bailiella, algunas especies poseen depresiones vinculares muy profundas y próximas al margen anterior, que podrían facilitar un enrollamiento pseudo-esferoidal, mientras que en otras el enrollamiento sería espiral. Por tanto, dicho carácter tiene entidad suficiente como para diferenciar ambos géneros. Además, el plectrum puede ser un carácter paedomórfico; en algunos pticopáridos se observa, en las últimas fases de meraspis, un plectrum encargado de unir la doblez del cefalón al hipostoma y que desaparece ya en los estados holaspis, y que marca el tránsito de formas con hipostomas fijos a flotantes (Zhang \& Jell, 1980, lám 80, fig. 1 n-p; Fortey, 1990, fig. 9), por lo que afianzaría más la separación de ambos géneros.

\section{Conclusiones}

El estudio del primer hallazgo de un ejemplar de Conocoryphe heberti enrollado junto con otro ejemplar incompleto y semienrollado de Occataria sdzuyi, ha permitido describir y analizar las estructuras coaptativas encontradas en estas dos especies, así mismo, se ha realizado un análisis en los cefalones y pigidios de Parabailiella languedocensis, Bailiella levyi y Bailiaspis souchoni, que ha permitido reconocer las mismas estructuras. Dicho análisis muestra la importancia de la morfología en las estructuras coaptativas a la hora de que los trilobites se enrollasen, bien de forma espiral o bien de forma esferoidal. A partir del análisis de las estructuras coaptativas de estas cinco especies de conocorífidos y teniendo en cuenta la variabilidad morfológica observada en los cefalones y pigidios que muestran, se plantea una hipótesis de cúal podría ser el tipo de enrollamiento que tendrían algunos taxones de conocorífidos, ya que la forma de enrollarse vendría determinada en gran medida por la morfología de las estructuras coaptativas presentes en el cefalón y el pigidio. De este modo se ha podido constatar que trilobites como Conocoryphe y Occatharia, con cranidios y pigidios muy similares, tendrían especies con un tipo de enrollamiento de esferoidal a pseudoesferoidal o espiral, y que en los casos de Bailiella, los cranidios y pigidios indican enrollamiento desde espiral a pseudoesferoidal y, por último, Bailiaspis y Parabailella que presentarían un enrollamiento exclusivamente espiral.

\section{AGRADECIMIENTOS}

Quiero agradecer al Prof. Eladio Liñán (Universidad de Zaragoza), con quien he discutido distintos puntos de este trabajo y cuyos comentarios críticos han mejorado el manuscrito original. También agradecer al Dr. Rodolfo Gozalo (Universidad de Valencia) su revisión crítica y todos sus valiosos comentarios, lo cuales han mejorando sustancialmente este trabajo. A las revisoras del manuscrito las Dras. Isabel Rábano (IGME) y María Dolores Gil Cid (UCM) por todos sus comentarios. A Jean Paul San-Martin y Didier Merle (Museo de Historia Natural de París) y a Raimond Faist y Susane Jiquel (Universite de Montperllier II) por facilitar el acceso al material depositado en sus respectivas instituciones. Agradecer a Isabel Pérez Urresti (Universidad de Zaragoza) por su ayuda técnica y fotográfica y a Fernando Gracia por su inestimable ayuda en el trabajo de campo.

Este trabajo es una contribución al proyecto Consolider CGL2006-12975/BTE («MURERO) del Ministerio de Educación y Ciencia de España; Grupo Consolidado E-17 y al Poyecto PM067/2006 del Gobierno de Aragón. Jorge Esteve disfruta de una beca de investigación pre-doctoral FPI.

\section{Referencias}

Álvaro, J.J. (2007). New ellipsocephalid trilobites from the lower Cambrian member of Láncara Formation, Cantabrian Mountains, northern Spain. Memoirs of the Association of Australasian Palaeontologist, 34: 343-355.

Álvaro, J.J. \& Vizcaïno, D. (1998). Revisión biostratigraphique du Cambrien moyen du versant méridional de la Montagne Noire (Languedoc, France). Bulletin de la Société Géologique de France, 2: 233-242.

Álvaro, J.J. \& Vizcaïno, D. (2003). The conocoryphid Biofacies: A bentic assemblage of normal-eyed and blind Trilobites. Special Paper in Palaeontology, 70: 127-140.

Babcock, L.E. \& Speyer, S.E. (1987). Enrolled trilobites from the Alden Pyrite Bed, Ledyard Shale (Middle Devonian) of Western New York. Journal of Paleonto$\log y, 61:$ 539-548.

Barrande, J. (1852). Systéme Silurien du Centre de la Boheme. $1^{\text {ere }}$ Partie. Crustacés. Trilobites. Praha. Paris. 935 pp.

Becq-Giraudon, J.F. \& Baillat, A. (2005). Eccaparadoxides (Trilobita) pouvaint s'enrouler. Un exemple rare 
du Cambrien moyen de Coulouma, Montagne Noire (Hérault, France). Bulletin de la Société Sciences Naturelle Ouest de la France, 27: 153-156.

Bergström, J. (1973). Organization, life and systematic of trilobites. Fossils and Strat., 2: 1-69.

Bruton, D.L. \& Haas W. (1997). Functional Morphology of Phacopinae (Trilobita) and the mechanics of Enrolment. Paleontographica Abt. A, 245: 1-43.

Bruton, D.L. \& Nakrem, H.A. (2005). Enrolment in a Middle Ordovician agnostoid trilobite. Acta Palaeontologica Polonica, 50: 441-448.

Chatterton, B.D. \& Campbell, M. (1993). Enrolling in trilobites: A review and some new characters. Association of Australasian Palaeontologist, 15: 103-123.

Clarkson, E.N.K. \& Henry, J.L. (1973). Structures coaptatives et enroulement chez quelques Trilobites ordoviciens et siluriens. Lethaia, 6: 105-132. doi:10.1111/j.1502-3931.1973.tb01186.x

Clarkson, E.N.K. \& Whittington, H. (1997). Enrollment and Coaptative Structures. In: Treatise on Invertebrate Paleontology Part 0, Revised. Arthropoda 1, Trilobita 1, (R.L. Kaesler, ed.) Geological Society of America-University of Kansas, Boulder and Lawrence, 67-74.

Cotton, T.J. (2001). The phylogeny and systematics of blind Cambrian Ptychoparioid trilobites. Palaeontology, 44: 167-207. doi:10.1111/1475-4983.00176

Courtessole, R. (1967). Une nouvelle espèce de Conocryphe «ocule» dans le Cambrien moyen du Nord-Leon. Bulletin de la Société Sciences Naturelle de Toulousse, 103: 491-526.

Courtessole, R. (1973). Le Cambrien Moyen de la Montagne Noire. Biostratigraphie. Imprim d'Oc, Toulouse, pp. 1-248.

Egovora, L.I.; Shabanov, Y.Y.; Pegel, V.E.; Savitsky, V.E.; Suchov, S.S. y Tchernysheva, N.E. (1982). The Mayan stage of the type locality (Middle Cambrian of the Siberian platform). Trudy Mezhvedomstvennyi Stratigraficheskii Komitet SSSR, 8: 1-146.

Esteve, J.; Zamora, S.; Gozalo, R. \& Liñán, E. (2008). Enrolment in Middle Cambrian Solenopleuropsinae Trilobites. Cuadernos del Museo Geominero, 9: 99-103.

Esteve, J.; Zamora, S.; Gozalo, R. \& Liñán, E. (en prensa). Sphaeroidal enrolment in middle Cambrian solenopleuropsine trilobites. Lethaia.

Fortey, R.A. (1990). Ontogeny, hypostome attachment and trilobite classification. Palaeontology, 33: 529-576.

Fortey, R.A. \& Owens, R.M. (1979). Enrollment in the classification of trilobites. Lethaia, 12: 219-226. doi:10.1111/j.1502-3931.1979.tb00998.x

Fortey, R.A. \& Hughes, N.C. (1998). Brood pouches in Trilobites. Journal of Paleontology, 72: 638-649.

Gil Cid, M.D. (1985). Sobre la existencia de Paradoxides enrollados en el Cámbrico hispánico. Boletín Geológico y Minero, 96: 15-17.

Gozalo, R. \& Liñán, E. (1988). Los materiales hercínicos de la Cordillera Ibérica en el contexto del Macizo Ibérico. Estudios Geológicos, 44: 399-404. doi:10.3989/egeol.88445-6556

Gozalo, R.; Liñán, E.; Palacios, T.; Gámez-Vintaned, J.A. \& Mayoral, E. (2003). The Cambrian of the Ibe- rian Peninsula: An overview. Geologica Acta, 1: 103-112.

Gozalo, R.; Liñán, E.; Gámez-Vintaned, J.A.; Dies, M.E.; Chirivella, J.B.; Zamora, S.; Esteve, J. \& Mayoral, E. (2008). The Cambrian of the Cadenas Ibéricas (NE Spain) and its Trilobites. Cuadernos del Museo Geominero, 9: 137-151.

Hammann, W. (1985). Life habit and enrolment in Calymenacea (Trilobita) and theirs significance for classification. Transactions of the Royal Society of Edinburgh, 76: 307-318.

Hammann, W. \& Rábano, I. (1987). Morphologie und Lebensweise der gattung Selenopeltis (Trilobita) und ihre Vorkommen im Ordovizium von Spanien. Senckenbergiana lethaea, 68: 91-137.

Henry, J.L. (1985). New information on the coaptative devices in the Ordovician trilobites Placoparia and Crozonaspis, and its significance for their classification and phylogeny. Transactions of the Royal Society of Edinburgh, 76: 319-324.

Hernández Sampelayo, P. (1935). Explicación del Nuevo Mapa Geológico de España. El sistema Cambriano. Memorias del Instituto Geológico y Minero de España, 1: 297-514.

Kim, D.H.; Westrop, S.R. \& Landing, E. (2002). Middle Cambrian (Acadian series) Conocoryphid and paradoxidid trilobites from the Upper Chamberlains brook Formation, Newfoundland and New Brunswick. Journal of Paleontology, 76: 822-842. doi:10.1666/00223360(2002)076<0822:MCASCA>2.0.CO;2

Korobov, M.N. (1973). Trilobites of the family Conocoryphidae from the Cambrian of the Siberia Platform and Tuva. Paleontologicheskiy Zhurnal, pp. 92-97.

Linnarsson, G. (1877). Om faunas I lagren med Paradoxides aelandicus. Geol. För. Förh., 3: 71-86.

Liñán, E. \& Gozalo, R. (1986). Trilobites del Cámbrico inferior y medio de Murero (Cordillera Ibérica). Memorias del Museo Paleontológico de la Universidad de Zaragoza, 2. 104 pp.

Liñán, E.; Perejón, A. and Sdzuy, K. (1993). The LowerMiddle Cambrian stages and stratotypes from the Iberian Peninsula: a revision. Geological Magazine, 130: 817-833. doi:10.1017/S0016756800023189

Liñán, E.; Villas, E.; Gámez Vintaned, J.A.; Álvaro, J.; Gozalo, R.; Palacios, T. \& Sdzuy, K. (1996). Síntesis Paleontológica del Cámbrico y Ordovícico del Sistema Ibérico (Cadenas Ibéricas y Cadenas Hespéricas). Revista Española de Paleontología, No extraordinario: $21-32$.

Liñán, E.; Gozalo, R.; Palacios, T.; Gámez Vinaned, J.A.; Ugidos, J.M. \& Mayoral, E. (2002). Cambrian. En: The Geology of Spain (Gibbons, W. \& Moreno, T., eds.). The Geological Society, London, 17-29.

Liñán, E.; Gozalo, R.; Dies, M.E.; Gámez Vintaned, J.A.; Mayoral, E.; Chirivilla, J.; Esteve, J.; Zamora, S.; Zhuravlev, A. Yu. \& Andrés, J.A. (2008). Lower and Middle Cambrian trilobites of selected localities in Cadenas Ibéricas (NE, Spain). Post-conference fieldtrip, Fourth International Trilobite Conference Trilo 08 Toledo, Spain, 1-52. 
Nedin, C. (1999). Anomalocaris predation on nonmineralized and mineralized trilobites. Geology, 27: 987-990. doi:10.1130/0091-7613(1999)027<0987:APO$\mathrm{NAM}>2.3 . \mathrm{CO} ; 2$

Öpik, A.A. (1967). The Midyallan fauna of noth-wetern Queensland. Bureau of Mineral Resources Australia Bulletin, 74(1): 1-404; (2): 1-167.

Robison, R.A. (1964). Late Middle Cambrian faunas from western Utah. Journal of Paleontology, 38: 510-566.

Sdzuy, K. (1961). Das Kambrium Spaniens. Teil II: Trilobiten. Akademie der Wissenschaften und der Literatur, Abhandlungen der mathematisch-naturwissenschaftlichen Klasse, 1961(7-8): 499-690 (217-408).

Sdzuy, K. (1968). Trilobites del Cámbrico Medio de Asturias. Trabajos de Geología, Universidad de Oviedo, año 1967, 1: 77-133.

Sdzuy, K. 1972. Das Kambrium der acadobaltischen Faunenprovinz. Zentralblatt für Geologie und Paläontologie, Teil II, 1972: 1-91.

Sdzuy, K. \& Liñán, E. (1996). Cornucoryphe schirmi n.gen. n. sp. an unusual conocoryphid trilobite from the Middle Cambrian of Spain. Palaontologische Zeitschrift, 70: 433-438.

Sdzuy, K.; Liñán, E. and Gozalo, R. (1999). The Leonian Stage (early Middle Cambrian): a unit for Cambrian correlation in the Mediterranean subprovince. Geological Magazine, 136: 39-48.

Šnajdr, M. (1958). Trilobiti českého středniho kambria (The trilobites of the Middle Cambrian of Bohemia). Nakl. ceského Akadmie Véd, Rozpravy Ustredního ústavu geologického, 24: 1-236.
Speyer, S.E. (1985). Enrolment strategies in Middle Devonian trilobites. Geological Society of America Abstracts with Programs, 17: 724.

Speyer, S.E. (1988). Biostratinomy and functional morphology of enrolment in two Middle Devonian trilobites. Lethaia, 51: 121-138. doi:10.1111/j.1502-3931.1988.tb00803.x

Stitt, J.H. (1983). Enrolled Late Cambrian Trilobites from the Davis Formation, Southeast Missouri. Journal of Paleontology, 57: 93-105.

Thoral, M. (1946). Conocoryphidæ Langudociens. Annales Université e Lyon, Sciences Naturelles, 5: 1-89.

Westergård, A.H. (1936). Paradoxides oelandicus beds of Öland with the account of diamond boring through the Cambrian at Mossberga. Sveriges Geologiska Undersökning Ser. C., 394: 1-66.

Whittington, H.B. (1987). Hypostomes and ventral cephalic sutures in Cambrian Trilobites. Palaeontology, 31: 577-609.

Zamarreño, I. (1972). Las litofacies carbonatadas del Cámbrico de la Zona Cantábrica (NO, España) y su distribución paleogeográfica. Trabajos de Geología, Universidad de Oviedo, 5: 1-118.

Zhang, W. \& Jell, P.A. (1987). Cambrian trilobites of North China - Chinese Cambrian trilobites housed in the Smithsonian Instititution. Science Press, Beijing, $332 \mathrm{pp}$.

Recibido el 16 de febrero de 2009

Aceptado el 24 de junio de 2009

Publicado online el 15 de diciembre de 2009 